\title{
DGPPN guideline on anxiety disorders and cognitive dysfunction in the elderly or patients with multiple sclerosis
}

\author{
Andrea Schmitt $^{1} \cdot$ Peter Falkai $^{1}$
}

Published online: 29 May 2015

(C) Springer-Verlag Berlin Heidelberg 2015

Anxiety disorders are among the most prevalent psychiatric conditions and include specific phobias as well as generalized anxiety disorder. Bandelow et al. [1] are reporting on the recently published DGPPN guidelines for the treatment of anxiety disorders in Germany. They are based on a systematic review of randomized controlled clinical trials on anxiety disorders and include recommendations for psychotherapeutic and pharmacological treatment strategies. The highest level of evidence had the cognitive behavioral therapy, followed by psychodynamic therapy. With respect to pharmacotherapy, selective serotonin reuptake inhibitors and serotonin-noradrenaline reuptake inhibitors are recommended. In the case of non-response, treatment should be switched to the other class of drugs or psychotherapy or a combination of both approaches. In a case-control study, based on a German population-based cross-sectional telephone survey, Kapfhammer et al. [2] found a lifetime prevalence of visual height intolerance of $28.5 \%$ and acrophobia of $6.4 \%$. Both anxiety disorders were associated with high rates of comorbid anxiety disorders and depression, leading to social impairment. Schoepf and Heun [3] address the relationship between anxiety disorders and physical comorbidity, especially hospital-based mortality during a 12.5-year observation period in seven General Manchester Hospitals. They found 22 physical comorbidities to be increased in patients with anxiety disorders, including cardiovascular diseases. The impact of atrial fibrillation, angina and gallbladder calculus on hospital-based mortality

Andrea Schmitt

Andrea.Schmitt@med.uni-muenchen.de

1 Department of Psychiatry and Psychotherapy, LudwigMaximilians-University Munich, Nußbaumstr. 7, 80336 Munich, Germany was higher in patients with anxiety disorders compared to controls. Psychosocial and physical comorbidities may be based on the interaction between genetic and environmental factors, e.g., influencing the serotonergic system. Gloster et al. [4] tested whether the 5HTT-LPR polymorphism influenced therapy response as a function of endophenotypes such as psychological flexibility, panic, agoraphobic avoidance and anxiety sensitivity in patients with panic disorder with agoraphobia. The 5HTT polymorphism status influenced therapy-related improvements in psychological flexibility, which may represent an important variable for the treatment of anxiety disorders.

Cognitive impairment is difficult to diagnose in the healthy elderly, even when using a neuropsychological test battery. Using the CERAD-NAB, Mistridis et al. [5] report that irrespective of cutoff criteria, high percentages of one or more abnormal scores were obtained. Below the tenth percentile, $60.0 \%$ of the normative sample obtained one or more scores. Therefore, in healthy individuals, the prevalence of low scores should be considered more cautiously to avoid false-positive diagnosis of mild cognitive impairment (MCI). The summary figure of CERAD-NAB base rates is an important supplement for test interpretation and can be used to improve diagnostic accuracy. Dementia prevention in the elderly is of major interest in clinical studies. In a longitudinal, multicenter study of 3327 community-dwelling persons aged at $<75$ years, Haenisch et al. [6] assessed the use of proton pump inhibitors, which are used for the treatment of gastrointestinal diseases and the risk of dementia in elderly people. During follow-up, 431 patients with any dementia and 260 patients with Alzheimer's disease were identified. Patients receiving proton pump inhibitors (PPIs) had a significant risk of any dementia and Alzheimer's disease compared to nonusers. Recently, it had been shown that PPIs potentially 
enhanced amyloid beta peptide $(A \beta)$ levels in brains of mice by affecting the enzymes $\beta$-secretase and $\gamma$-secretase. In the differential diagnosis of dementia, potentially reversible conditions with neurocognitive impairment have to be carefully assessed. Using routine laboratory parameters and neuroimaging in a retrospective study in 166 patients with suspected dementia, Djukic et al. [7] found 99 patients with already known dementia. In this subgroup, authors found a potentially treatable cause in $18.2 \%$. In contrast, in the newly diagnosed patients, $31.1 \%$ prevailed potentially reversible causes of dementia. The most common cases were depressive pseudodementia, followed by vitamin B12 deficiency, chronic alcoholism, normal pressure hydrocephalus endocrine diseases and possibly abnormally reduced folate concentration in serum.

In patients with multiple sclerosis (MS), cognitive impairment has an impact on everyday functioning and quality of life. Adler and Lembach [8] for the first time applied a computer-based method, the memory and attention test (MAT) in 531 outpatients with MS compared to 84 healthy probands. In MS patients compared to healthy controls, episodic short-term memory was significantly decreased and negatively correlated with the Expanded Disability Status Scale (EDSS) score. In addition, verbal short-term memory, episodic working memory and selective attention were impaired in patients with MS and may contribute to psychosocial disabilities and burden of disease.

\section{References}

1. Bandelow B, Lichte T, Rudolf S, Wiltink J, Beutel ME (2014) The German guidelines for the treatment of anxiety disorders. Eur Arch Psychiatry Clin Neurosci. doi:10.1007/ s00406-014-0563-Z

2. Kapfhammer H-P, Huppert D, Grill E, Fitz W, Brandt T (2014) Visual height intolerance and acrophobia: clinical characteristics and comorbidity patterns. Eur Arch Psychiatry Clin Neurosci. doi:10.1007/s00406-014-0548-y

3. Schoepf D, Heun R (2014) Anxiety disorders and physical comorbidity: increased prevalence but reduced relevance of specific risk factors for hospital-based mortality during a 12.5-year observation period in general hospital admissions. Eur Arch Psychiatry Clin Neurosci. doi:10.1007/s00406-014-0566-9

4. Gloster AT, Gerlach AL, Hamm A., Höfler M, Alpers GW, Kircher T, Reif A (2015) 5HTT is associated with the phenotype psychological flexibility: results from a randomized clinical trial. Eur Arch Psychiatry Clin Neurosci. doi:10.1007/ s00406-015-0575-3

5. Mistridis P, Egli SC, Iverson GL, Berres M, Willmes K, WelshBohmer KA., Monsch AU (2015) Considering the base rates of low performance in cognitively healthy older adults improves the accuracy to identify neurocognitive impairment with the consortium to establish a registry for Alzheimer's disease-neuropsychological assessment battery (CERAD-NAB). Eur Arch Psychiatry Clin Neurosci. doi:10.1007/s00406-014-0571-z

6. Haenisch B, von Holt K, Wiese B, Prokein J, Lange C, Ernst A., Scherer M (2014) Risk of dementia in elderly patients with the use of proton pump inhibitors. Eur Arch Psychiatry Clin Neurosci. doi:10.1007/s00406-014-0554-0

7. Djukic M, Wedekind D, Franz A., Gremke M, Nau R (2015) Frequency of dementia syndromes with a potentially treatable cause in geriatric in-patients: analysis of a 1-year interval. Eur Arch Psychiatry Clin Neurosci. doi:10.1007/s00406-015-0583-3

8. Adler G, Lembach Y (2015) Memory and selective attention in multiple sclerosis: cross-sectional computer-based assessment in a large outpatient sample. Eur Arch Psychiatry Clin Neurosci. doi:10.1007/s00406-015-0574-4 